\title{
Spray dried cubosomes with ovalbumin and Quil-A as a nanoparticulate dry powder vaccine formulation
}

von Halling Laier, Christoffer; Gibson, Blake; van de Weert, Marco; Boyd, Ben J.; Rades, Thomas; Boisen, Anja; Hook, Sarah; Nielsen, Line Hagner

Published in:

International Journal of Pharmaceutics

Link to article, DOI:

10.1016/j.ijpharm.2018.08.036

Publication date:

2018

Document Version

Peer reviewed version

Link back to DTU Orbit

Citation (APA):

von Halling Laier, C., Gibson, B., van de Weert, M., Boyd, B. J., Rades, T., Boisen, A., Hook, S., \& Nielsen, L. H. (2018). Spray dried cubosomes with ovalbumin and Quil-A as a nanoparticulate dry powder vaccine formulation. International Journal of Pharmaceutics, 550(1-2), 35-44. https://doi.org/10.1016/j.ijpharm.2018.08.036

\section{General rights}

Copyright and moral rights for the publications made accessible in the public portal are retained by the authors and/or other copyright owners and it is a condition of accessing publications that users recognise and abide by the legal requirements associated with these rights.

- Users may download and print one copy of any publication from the public portal for the purpose of private study or research.

- You may not further distribute the material or use it for any profit-making activity or commercial gain

- You may freely distribute the URL identifying the publication in the public portal 


\section{Spray dried cubosomes with ovalbumin and Quil-A as a nanoparticulate dry powder vaccine formulation}

Christoffer von Halling Laier ${ }^{a^{*}}$, Blake Gibson ${ }^{b}$, Marco van de Weert ${ }^{c}$, Ben J. Boyd ${ }^{d}$, Thomas Rades ${ }^{c, e}$, Anja Boisen ${ }^{\mathrm{a}}$, Sarah Hook ${ }^{\mathrm{b}}$, Line Hagner Nielsen ${ }^{\mathrm{a}}$

${ }^{a}$ Department of Micro- and Nanotechnology, Technical University of Denmark, Oersteds Plads 345C, 2800 Kgs. Lyngby, Denmark

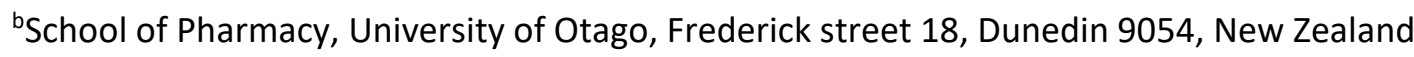

'Department of Pharmacy, Faculty of Health and Medical Sciences, University of Copenhagen, Universitetsparken 2, 2100 Copenhagen, Denmark

${ }^{\mathrm{d} D r u g}$ Delivery, Disposition and Dynamics, and ARC Centre of Excellence in Convergent Bio-Nano Science and Technology, Monash Institute of Pharmaceutical Sciences, Monash University (Parkville Campus), 381 Royal Parade, Parkville, VIC 3052, Australia

'Faculty of Science and Engineering, Åbo Akademi University, Tykistökatu 6A, 20521 Turku, Finland

\footnotetext{
* Corresponding author: Department of Micro- and Nanotechnology, Technical University of Denmark, Oersteds Plads 345C, 2800 Kgs. Lyngby, Denmark.

Phone: +45 452557 87, E-mail: cvohal@nanotech.dtu.dk (C. von Halling Laier)
} 


\begin{abstract}
Subunit vaccine formulations are often produced as liquid dispersions through complicated processes. It is desirable, however, to have simple, cheap and up-scalable methods to produce nanoparticulate subunit vaccines in powder form. Here, a simple single-step spray drying process for production of powder cubosome precursors with the model antigen ovalbumin (OVA) and the adjuvant Quil-A is presented. The cubosomes were characterized in vitro and evaluated in vivo by subcutaneous and oral administration for their potential as a vaccine formulation. Hydrated cubosomes had average particle size of $257 \pm 8 \mathrm{~nm}$ and zeta potential of $-18.0 \pm 0.6 \mathrm{mV}$. The powder contained $10.6 \pm 0.7 \% \mathrm{w} / \mathrm{w}$ OVA prior to hydration, of which

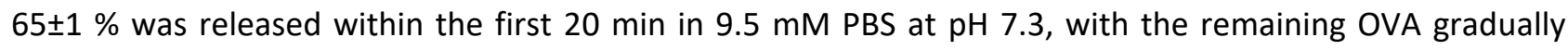
released over the following $24 \mathrm{~h}$. Immunization with cubosomes resulted in significantly stronger antigenspecific serum IgG responses $(p<0.01), C D 8^{+} T$ cell expansion $(p<0.0001)$ and target $T$ cell killing compared to controls when given s.c., and was ineffective orally. This study shows that spray drying is a suitable method for producing nanoparticulate vaccine formulations in dry powder form.
\end{abstract}




\section{Introduction}

Immunization through vaccination is the most significant and cost-effective medical contribution to public health and disease prevention (Azmi et al., 2014; Foged, 2011). However, many pathogens remain the cause of serious health issues in large areas of the world, thus sustaining the need for continued vaccine development and administration (Ehreth, 2003; Foged, 2011; Zhao and Leong, 1996). An important limitation to the worldwide administration of vaccines is the high costs of manufacturing and distribution. Consequently, there is an additional need for developing inexpensive methods of producing vaccines which can be distributed at reduced costs (Hill et al., 2016). Vaccines have traditionally been based on liveattenuated or whole-killed organisms. These have intrinsic immunostimulatory capacity, sufficient to elicit a strong immune response. However, due to the present emphasis on safety in vaccines, modern vaccines are based on highly purified peptides or proteins referred to as subunit antigens (Azmi et al., 2014; Foged, 2011). Subunit antigens lack the secondary signals required to elicit a strong immune response and must therefore be formulated with adjuvants. Adjuvants are compounds that are delivered together with antigens to improve and modulate the immune response to a vaccine (Azmi et al., 2014; Foged, 2011).

The protein ovalbumin (OVA) is often used as a model antigen as it is relatively inexpensive and accessible. A wide range of immunopotentiating compounds exist, including bacterial products, aluminum salts, immunopotentiating cytokines and natural products such as Quil-A. Quil-A is an inexpensive adjuvant obtained by purification of an extract from the bark of the Chilean tree Quillaja saponaria Molina (Kensil et al., 1995). It is a Toll-like receptor independent adjuvant composed of a heterogeneous mixture of chemically related triterpenoid saponins (Hook and Rades, 2013; Kensil et al., 1995) and has been studied widely for use in immune stimulating complexes (Hook and Rades, 2013) or together with delivery systems such as liposomes (Demana et al., 2004; Gordon et al., 2012; Rattanapak et al., 2012; White et al., 2006) and cubosomes (Gordon et al., 2012; Kojarunchitt et al., 2015; Rattanapak et al., 2012). Quil-A can induce strong antibody responses and cytotoxic $T$ cell responses. Cytotoxic $T$ cell responses are necessary for protection against intracellular pathogens and in the removal of infected or transformed cells and are often difficult to achieve with non-replicating vaccines (Hook and Rades, 2013; Myschik et al., 2008a; White et al., 2006). Saponins are able to associate with cholesterol in cell membranes and create membrane pores. This is proposed to be the principal mechanism for their potentiation of immune responses, although several other mechanisms have also been suggested (Gordon et al., 2012; Hook and Rades, 2013; Kersten and Crommelin, 2003). Pore formation may allow vaccines to enter the cytosol of antigen presenting cells (APCs) thus allowing for cross-presentation of antigen and facilitating cytotoxic $\mathrm{T}$ cell mediated immunity (Heeg et al., 1991).

Particulate subunit vaccine delivery systems are inherently more immunogenic than their soluble counterparts. This has commonly been attributed to an ability to protect the antigen from rapid degradation, prolonged antigen presentation, better uptake by antigen presenting cells and physical similarity to native pathogens (Foged, 2011; Storni et al., 2005). A wide range of micro- and nano-sized colloidal particles have been investigated as delivery systems including polymeric and lipid based particles such as emulsions and liposomes (Foged, 2011). Liposomes are dispersions of lamellar liquid crystals (lamellar mesophases) forming spherical particles with a lipid bilayer surrounding an aqueous core (Foged, 2011; Rizwan et al., 2013). This gives liposomes the ability to encapsulate a wide range of bioactive 
compounds, and liposomes have therefore been studied extensively for vaccine delivery purposes (Foged, 2011; Rizwan et al., 2013).

Analogous to liposomes, cubosomes are dispersions of the bicontinuous cubic mesophase (Rizwan et al., 2013). The basic unit of the bicontinuous cubic mesophase is a lipid bilayer, which is contorted into an isotropic infinite periodic minimal surface (IPMS) with 3-dimensional long-range order (Hyde, 2001; Spicer et al., 2002a). Like the bulk cubic phase, cubosomes are composed of a highly contorted lipid bilayer forming a continuous lipid domain and two highly tortuous but non-intersecting water domains. Cubosomes therefore have a high lipid content and a large surface area giving them the ability to encapsulate a wide range of bioactive compounds (Rizwan et al., 2007; Spicer, 2005). This makes cubosomes flexible with regards to the types of antigens and adjuvants they can carry. Rizwan et al. have shown that cubosomes allow for a larger amount of encapsulated compound compared to liposomes and also provide a more efficient retention of an antigen (Rizwan et al., 2013). Furthermore, cubosomes have been shown in vivo to be a promising particulate carrier for vaccines as they effectively promote antigen transport into the draining lymph nodes (Liu et al., 2016) and are capable of stimulating a stronger immune response than equally adjuvanted liposomes (Rizwan et al., 2013).

Cubosomes are traditionally produced by a top-down approach in which a bulk liquid crystal is mixed with polymeric stabilizers in water and disrupted into nanoparticles by use of high-energy input fragmentation (Caltagirone et al., 2014; Rizwan et al., 2011; Uyama et al., 2009). These preparation methods are laborious and difficult to scale up (Rizwan et al., 2011) and therefore alternative methods for preparing cubosomes are highly desirable. The bottom-up approach exploits self-assembly of certain lipids dissolved in organic solvent upon dilution in excess water (Rizwan et al., 2011; Spicer, 2003). This approach is simple, easy to scale up and avoids the need for high-energy input. However, it can additionally be desirable to have the particles in a powder form, which spontaneously forms cubosomes upon rehydration (Nielsen et al., 2017; Spicer et al., 2002a). These powders are often referred to as 'precursors' and have earlier been produced by spray drying (Avachat and Parpani, 2015; Nielsen et al., 2017; Shah et al., 2006; Spicer et al., 2002b) or freeze-drying (Bei et al., 2010). Spray dried powder forms of vaccine formulations are easy to transport and can provide improved storage stability. They thereby hold the promise to circumvent the need for cold-chain storage, which is necessary for the majority of available vaccine formulations in order to maintain antigen integrity (Sou et al., 2011). Ohtake et al. reported the successful use of spray drying to produce a dry powder formulation of a measles vaccine. Here, the storage stability of the powder was improved to 8 weeks at $37^{\circ} \mathrm{C}$ compared to 1 week for the commercial lyophilized formulation (Ohtake et al., 2010).

Spray drying is a method in which a highly dispersed liquid is brought into contact with a sufficient amount of hot air to dry the droplets into particles through evaporation (Ameri and Maa, 2006; Fang and Bhandari, 2017). It has the advantage of being a highly versatile, cheap and scalable method and can be operated under aseptic conditions (Fang and Bhandari, 2017; Searles and Cherian, 2015). Furthermore, it is well suited for drying labile products, as can be essential when producing vaccine formulations (Ameri and Maa, 2006; Fang and Bhandari, 2017). In the literature, it is reported that spray drying molten monoolein together with aqueous starch resulted in powders, but the mixture was difficult to spray dry as a cubic phase is immediately formed upon hydration of monoolein (Spicer et al., 2002a). To solve the problem, 
Spicer et al. added ethanol as a hydrotrope to dissolve the cubic phase and substituted starch with the ethanol-soluble dextran, thus obtaining an emulsion which could easily be spray dried. The addition of dextran provided a flowable spray dried powder and helped stabilize the colloidal cubosomes that formed spontaneously upon hydration of the powder (Spicer et al., 2002a). In later work, a similar method was used to spray dry cubosomes with the incorporation of the protein OVA (Nielsen et al., 2017). However, an adjuvant is required in order to obtain a complete vaccine formulation, and was not included in that study.

Cubosomes have previously been studied for their use in parenteral vaccines (Gordon et al., 2012; Kojarunchitt et al., 2015; Liu et al., 2016; Rizwan et al., 2013) but their use in oral vaccines has not been reported due to concerns of limited stability in the gastro intestinal tract and the induction of an adequate immune response. However, they are an appealing option for oral delivery of antigens as cubic phase liquid crystals made from monoolein are reported to be biocompatible, biodegradable, bioadhesive (Nielsen et al., 1998; Patton and Carey, 1979) and able to increase the oral bioavailability of poorly soluble drugs (Ali et al., 2017). Oral vaccines are desirable for compliance, but more importantly aid mass vaccination by easing logistics and avoiding needles, thus eliminating the need for highly trained personnel and improving safety especially in developing countries (Azizi et al., 2010; Gebril et al., 2012).

The aim of this study was to provide a simple and scalable method for producing a dry powder subunit protein vaccine formulation by spray drying. Cubosomes containing OVA as a model antigen and the adjuvant Quil-A were produced, characterized in vitro and then evaluated in vivo in mice by measuring humoral and cellular immune responses following subcutaneous (s.c.) and oral delivery.

\section{Materials and methods}

\section{Materials}

$\operatorname{Dimodan}^{\circ} \mathrm{MO}$ 90/D (monoolein) was kindly donated by Danisco (Grindsted, Denmark). Fluorescein isothiocyanate (FITC - isomer I), ovalbumin (Grade VII, from chicken egg white) and dextran (from Leuconostoc spp., $40 \mathrm{kDa}$ ) were purchased from Sigma-Aldrich (St. Louis, MO, USA). Quil-A was obtained from Brenntag Biosector (Frederikssund, Denmark) and phosphate buffered saline (PBS) tablets were acquired from Oxoid limited (Basingstoke, England). 5,6-carboxyfluorescein diacetate succinimidyl ester (CFSE) and CellTrace ${ }^{\mathrm{TM}}$ Violet Cell Proliferation Kit (CTV) were purchased from Molecular Probes (Eugene, OR, USA). Alum (Alu-Gel-S) was purchased from Serva (Heidelberg, Germany). OVA $257-264$ peptide (SIINFEKL) was purchased from Mimotopes (Clayton, Australia). Milli-Q water (Merck Millipore, Darmstadt, Germany) was used throughout the study. All other chemicals were of analytical grade and used as received. $100 \mathrm{kDa}$ ultrafiltration filters (Amicon Ultra-0.5 with Ultracell 100 membrane) were purchased from Merck Millipore (Darmstad, Germany), and Hellma absorption cuvettes (Suprasil ${ }^{\circ}$ quartz, $1 \mathrm{~mm}$ path length) were purchased from Sigma-Aldrich (St. Louis, MO, USA).

\section{Mice}

Specific pathogen free 6-8 weeks old male C57BL/6 (B6), OT-I and OT-II mice were obtained from the Hercus Taieri Resource Unit, University of Otago, Dunedin, New Zealand. Mice had unlimited access to food and water throughout the experiment. All experiments were approved by the Animal Ethics Committee at the University of Otago (AEC no. 80-16). 


\section{Spray drying of cubosome precursors containing OVA and Quil-A}

Cubosome precursors were prepared using monoolein (Dimodan ${ }^{\circ} \mathrm{MO} 90 / \mathrm{D}$ ), and the cubosomes were loaded with OVA and Quil-A and stabilized by dextran. Dimodan was dissolved in ethanol $(5.33 \mathrm{mg} / \mathrm{mL})$ and then mixed 1:3.04 (v/v) with a solution of OVA, Quil-A and dextran in water $(0.52,0.035$ and $2.63 \mathrm{mg} / \mathrm{mL}$, respectively). The resulting emulsion was stirred for $15 \mathrm{~min}$ and then spray dried using a Büchi B-290 mini spray dryer (Büchi Labortechnik AG, Flawil, Switzerland) in open-loop configuration with a B-296 dehumidifier (Büchi Labortechnik AG, Flawil, Switzerland) and a pressure nozzle with a diameter of $1.5 \mathrm{~mm}$. Air was utilized as drying gas with the spray drying parameters given in Table 1. Blank particles with dextran as well as particles with dextran and either OVA or Quil-A were produced to be used as controls. Following completion of the process, spray dried powders were collected and stored in glass vials. Residual moisture in the powders was further reduced in a secondary drying step by placing the powders in an oven at $86 \pm 2^{\circ} \mathrm{C}$ for $24 \mathrm{~h}$. Afterwards, the powders were stored in a dry environment.

Table 1. Spray drying parameters applied for spray drying of cubosomes

\begin{tabular}{|l|l|}
\hline Feed rate & $4.5 \mathrm{~mL} / \mathrm{min}$ \\
\hline Atomizing air flow & $667 \mathrm{~L} / \mathrm{h}$ \\
\hline Aspirator rate & $100 \%$ \\
\hline Inlet temperature & $150^{\circ} \mathrm{C}$ \\
\hline Measured outlet temperature & $86^{\circ} \mathrm{C}$ \\
\hline
\end{tabular}

\section{Internal structure and morphology of cubosomes using SAXS and Cryo-TEM}

The internal structure of hydrated particles dispersed in water was investigated by small angle X-ray scattering (SAXS) at the SAXS/WAXS beamline at the Australian Synchrotron, Clayton, Australia (Kirby et al., 2013). Powders were reconstituted at $50 \mathrm{mg} / \mathrm{mL}$ in water using vortex mixing together with four cycles of 3 min ultrasonication with $2 \mathrm{~mm}$ solid glass beads and then loaded into glass capillaries. The X-ray beam had an energy of $12 \mathrm{keV}(1.03 \AA)$ and the sample was placed $1531 \mathrm{~mm}$ from the detector. Diffraction patterns were converted to intensity vs q-value plots to enable the identification of relative peak positions and corresponding Miller indices, which were then used to identify the space group of the dominant internal nanostructure of the sample (Hyde, 2001). The internal structure of dry particles prior to hydration was investigated in a similar manner at the Austrian SAXS/WAXS beamline at the synchrotron light source ELETTRA (Trieste, Italy).

The morphology of the hydrated particles containing OVA and Quil-A was further investigated by cryogenic transmission electron microscopy (cryo-TEM) using a Tecnai G2 20 TWIN transmission electron microscope (FEl, Hillsboro, OR, USA). Particles were reconstituted at $5 \mathrm{mg} / \mathrm{mL}$ in water and then prepared using a FEI Vitrobot Mark IV under controlled $\left(4^{\circ} \mathrm{C}, 100 \%\right.$ relative humidity) and automated conditions (blot time $3 \mathrm{~s}$, blot force 0). $5 \mu \mathrm{L}$ of sample was deposited on a glow-discharged 300 mesh lacey carbon grid (Pelco Lacey, Ted Pella Inc., CA, USA). Excess liquid was removed by blotting and the sample was then immediately snap frozen in liquid ethane and kept in liquid nitrogen. Imaging was performed at an acceleration voltage of $200 \mathrm{kV}$ with a $4 \mathrm{~K}$ CCD Eagle digital camera from FEI. 


\section{Size, zeta potential and stability of cubosomes}

The average particle size (z-average) of hydrated particles was measured by dynamic light scattering (DLS) using the photon correlation spectroscopy technique and the zeta potential of the particles was investigated using the laser-Doppler electrophoresis method. All measurements were performed using a Zetasizer Nano Zs (Malvern Zetasizer, NanoZs Zen 3600, Malvern, UK) equipped with a $633 \mathrm{~nm}$ laser and $173^{\circ}$ detection optics at $25^{\circ} \mathrm{C}$. Data acquisition and analysis was performed using Malvern DTS v.7.12 software (Malvern, UK). For size measurements, $1 \mathrm{mg} / \mathrm{mL}$ of powders were reconstituted in filtered $9.5 \mathrm{mM}$ PBS at pH 7.3 using four cycles of 3 min ultrasonication with glass beads and then measured by DLS after 15 min. Colloidal stability was investigated by storing the particles at $25^{\circ} \mathrm{C}$ and measuring particle size after $0.5,6$ and $24 \mathrm{~h}$. For zeta potential measurements, powders were reconstituted at $1 \mathrm{mg} / \mathrm{mL}$ in the same manner as for size measurements, but using a filtered $9.5 \mathrm{mM}$ phosphate buffer at $\mathrm{pH}$ 7.3. All measurements were performed in triplicates of three independent samples. Morphological stability of cubosomes with OVA and Quil-A was investigated by periodic SAXS measurements for $26 \mathrm{~h}$ of a $50 \mathrm{mg} / \mathrm{mL}$ cubosomes suspension in PBS at $37^{\circ} \mathrm{C}$ as described above.

\section{OVA load, encapsulation efficiency and release of OVA from cubosomes}

FITC was conjugated to OVA as described by Könnings et al. (Könnings et al., 2002) and cubosome precursors containing FITC-OVA and Quil-A were produced as described above. The load of FITC-OVA in the powder was measured by dissolving $0.25 \mathrm{mg} / \mathrm{mL}$ of FITC-OVA and Quil-A containing cubosomes in $5 \% \mathrm{v} / \mathrm{v}$ Triton X-100 in 9.5 mM PBS at pH 7.3 immediately before measurements. Standards of FITC-OVA spiked into $0.25 \mathrm{mg} / \mathrm{mL}$ dissolved Quil-A cubosomes were prepared in the same way as the samples. The standards and samples were analyzed by spectrofluorimetry on a Varioskan ${ }^{\mathrm{TM}}$ LUX microplate reader (ThermoFisher Scientific, Denmark) using 494 and $520 \mathrm{~nm}$ as excitation and emission wavelength, respectively, and $500 \mathrm{~ms}$ of collection time. Standards and samples were prepared in three independent replicates with four repeated aliquots of each.

Encapsulation efficiency and release of OVA from the cubosomes were measured using an ultra-filtration method. FITC-OVA and Quil-A containing cubosomes were rehydrated at $0.2 \mathrm{mg} / \mathrm{mL}$ in $9.5 \mathrm{mM}$ PBS at pH 7.3 using four cycles of 3 min ultrasonication with glass beads. Hydrated cubosomes were kept rotating at a speed of $150 \mathrm{rpm}$ at $37^{\circ} \mathrm{C}$ in the dark. Aliquots from four independent samples were taken at $0,0.5,1,2,4$, $6,8,24,27,30$ and $48 \mathrm{~h}$ after hydration and immediately subjected to ultrafiltration through a $100 \mathrm{kDa}$ membrane (allowing FITC-OVA to pass but retaining the cubosomes), facilitated by centrifugation at 14,000 $\mathrm{G}$ for $10 \mathrm{~min}$. Filtrates were then collected and stored in the dark until fluorimetric analysis was performed in the same way as described above for antigen load. The $0 \mathrm{~h}$ time point was used to estimate the entrapment of FITC-OVA in the cubosomes. Three independent sets of standards were prepared by spiking FITC-OVA into $0.2 \mathrm{mg} / \mathrm{mL}$ Quil-A cubosomes in $9.5 \mathrm{mM} \mathrm{PBS,} \mathrm{pH} \mathrm{7.3,} \mathrm{followed} \mathrm{by} \mathrm{ultrafiltration} \mathrm{similar} \mathrm{to} \mathrm{the}$ loaded cubosomes.

\section{Stability of secondary structure of OVA during formulation and storage}

Changes in secondary structure of OVA during cubosome production were investigated by circular dichroism (CD). Far-UV CD spectra $(190-260 \mathrm{~nm})$ were recorded at $20^{\circ} \mathrm{C}$ in a $1 \mathrm{~mm}$ quartz cuvette on a Chirascan CD spectrometer (Applied Photophysics, Leatherhead, UK) equipped with a Peltier thermostated cell holder. Ten scans were averaged at $0.5 \mathrm{~nm}$ step-size and $0.5 \mathrm{~s}$ integration time per point. 
Cubosomes were reconstituted to $1 \mathrm{mg} / \mathrm{mL}$ with vortex mixing and ultrasonication (as described above) in $9.5 \mathrm{mM}$ phosphate buffer, $\mathrm{pH}$ 7.3. Cubosome samples from two different batches were measured before and after the secondary drying step $\left(24 \mathrm{~h}\right.$ at $\left.86^{\circ} \mathrm{C}\right)$. For investigation of stability of OVA during storage, the CD spectrum was recorded for a sample of OVA and Quil-A cubosomes which had been stored for 6 months in dry conditions at room temperature. CD spectra of Quil-A containing cubosomes were subtracted as background from the spectra from OVA and Quil-A containing cubosomes. OVA in $9.5 \mathrm{mM}$ phosphate buffer at $\mathrm{pH} 7.3$ was used as a control for the native protein and OVA boiled for $2 \mathrm{~h}$ was used as a control for disruption of secondary structure. Lyophilized OVA was heated to $86 \pm 2^{\circ} \mathrm{C}$ for $24 \mathrm{~h}$ and used as control for whether the cubosomes stabilize the OVA during the secondary drying step. In all samples, OVA was used in a concentration of $0.106 \mathrm{mg} / \mathrm{mL}$.

\section{Immunological Studies}

Cubosomes were stored for 1-2 months at room temperature prior to use for the vaccine study. $\mathrm{C} 57 \mathrm{BI} / 6$ mice received $2 \times 10^{6}$ naïve OT-I and OT-II lymphocytes (which have T cell receptors that recognize CD8 and CD4 epitopes from OVA (Kojarunchitt et al., 2015)) in $200 \mu \mathrm{L}$ PBS injected intravenously (i.v.) into the tail vein on day -1 (Figure 1). Oral groups received vaccine on days 0,14 and 28 while s.c. groups received vaccine injected into the flank on days 14 and 28. Oral vaccines consisted of spray dried cubosomes with $100 \mu \mathrm{g}$ OVA and $6.7 \mu \mathrm{g}$ Quil-A in a capsule (Torpac size M) or a solution of $100 \mu \mathrm{g}$ OVA and $6.7 \mu \mathrm{g}$ Quil-A in $100 \mu \mathrm{L}$ PBS. Subcutaneous vaccines used were a solution of cubosomes with $0.67 \mu \mathrm{g}$ Quil-A in $200 \mu \mathrm{L}$ PBS (no antigen), a solution of $10 \mu \mathrm{g}$ OVA and $0.67 \mu \mathrm{g}$ Quil-A either unformulated or in cubosomes in $200 \mu \mathrm{L}$ PBS, or $10 \mu \mathrm{g}$ OVA in $200 \mu \mathrm{L}$ alum.

Four days after the last immunization, mice were injected i.v. with $4 \times 10^{6} \mathrm{C} 57 \mathrm{BI} / 6$ lymphocytes pulsed with $10 \mu \mathrm{g} / \mathrm{mL}$ SIINFEKL and labelled with CFSE in combination with $4 \times 10^{6}$ unpulsed lymphocytes labelled with CTV. Mice were sacrificed on day 34 and blood, spleens and lymph nodes were collected.

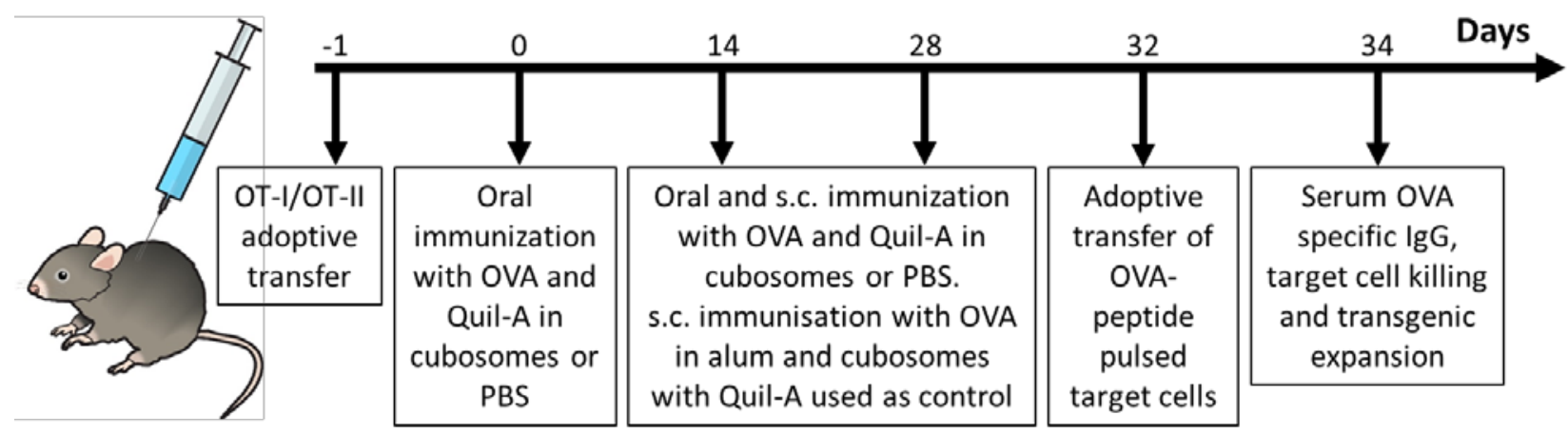

Figure 1. Timeline of the in vivo experiment investigating the immune stimulating effects of spray dried cubosomes with OVA and Quil-A.

\section{Flow cytometry}

Individual spleens and pooled lymph nodes were processed into single cell suspensions essentially as previously described (Myschik et al., 2008a). Aliquots of cells were stained with anti-CD8 (BioLegend ${ }^{\circ}$ ), anti$\mathrm{CD} 4$, anti- $\mathrm{V}_{\alpha} 2$ and anti- $\mathrm{V}_{\beta} 5$ antibodies (BD Pharmingen ${ }^{\mathrm{TM}}$ ) and propidium iodide (BioLegend ${ }^{\circ}$ ). Data was acquired on a BD FACSCanto ${ }^{\mathrm{TM}} \|$ (BD Biosciences) and was analyzed using FlowJo version 10.3 software 
(Tree Star, Inc.) using the gating strategy shown in Figure S1. The antigen-specific killing of peptide pulsed target cells was calculated as

$$
\% \text { killing }=\left(1-\frac{\text { peptide pulsed cells }}{\text { unpulsed cells }}\right) \times 100 \%
$$

and normalized to the negative control (cubosomes with Quil-A but without OVA) as $0 \%$.

\section{Serum IgG levels in vaccinated mice}

Blood samples were left overnight at $4^{\circ} \mathrm{C}$ to allow clotting and sera were separated by centrifugation at 9,402 G for $10 \mathrm{~min}$. OVA specific serum IgG was measured by ELISA. High-binding 96 well plates (Corning inc. Corning, NY, USA) were coated overnight at $4^{\circ} \mathrm{C}$ with $100 \mu \mathrm{L}$ of $10 \mu \mathrm{g} / \mathrm{mL}$ OVA in $50 \mathrm{mM}$ carbonate buffer at pH 9.6. Wells were blocked with $150 \mu$ L blocking buffer (PBS pH 7.3 with $2 \% \mathrm{w} / \mathrm{v}$ BSA) for $1 \mathrm{~h}$ at room temperature and washed 6 times with wash buffer (PBS pH 7.3 with $0.05 \% \mathrm{v} / \mathrm{v}$ Tween-20). Serum samples (diluted 1:100) were added to the first column of well plates, serially diluted across the plate and incubated for $2 \mathrm{~h}$ at room temperature. The plates were washed and 100 of $225 \mathrm{ng} / \mathrm{mL} \mathrm{HRP} \mathrm{Goat} \mathrm{anti-}$ mouse IgG (BioLegend ${ }^{\circ}$ ) was added to wells and incubated for $1 \mathrm{~h}$ at room temperature. Plates were washed and $100 \mu \mathrm{L}$ of substrate reagent solution (Substrate reagent pack, R\&D SYSTEMS ${ }^{\circledR}, M N, U S A$ ) was added and incubated at room temperature for $5 \mathrm{~min}$. The reaction was then stopped with $50 \mu \mathrm{L}$ of $2 \mathrm{M}$ $\mathrm{H}_{2} \mathrm{SO}_{4}$ and the absorbance was read at $450 \mathrm{~nm}$ using a Polarstar Omega Microplate Reader (BMG Labtech, Ortenberg, Germany).

\section{Statistics}

All statistical analysis was performed with Prism 7 (GraphPad, San Diego, USA). The experiments were performed in 3-4 replicates and the data is presented as mean \pm standard deviation (SD). Where relevant, statistical significance was determined by unpaired two-sided T-tests, using a significance level of $5 \%$ or by ordinary one-way ANOVA and Tukey's multiple comparisons test.

\section{Results and discussion}

\section{Spray drying of cubosome precursors containing OVA and Quil-A}

One of the best characterized binary systems forming cubic mesophases is the monoolein/water system. This system is desirable to use for cubosome production due to the resulting temperature stability and the availability of low-cost raw materials (Spicer et al., 2001). Phytantriol has recently been used increasingly as cubic phase forming lipid because it offers increased stability and purity, but monoolein was preferred here as it is substantially cheaper (Rizwan et al., 2013, 2011).

In this study, powder cubosome precursors containing OVA and/or Quil-A as well as blank particles were produced by mixing together solutions of monoolein and dextran with OVA and Quil-A added as applicable, followed by spray drying the mixture to obtain a powder. Preliminary studies showed that a monoolein to dextran weight ratio of 2:3 provided good stabilization of the spray dried powder without adding unnecessary extra bulk. Powder yields from the spray drying process were approximately $75 \%$ for all the formulations and white opaque dispersions resulted from rehydration of the powders. 


\section{Internal structure and morphology of cubosomes}

The monoolein/water system gives rise to two main forms of the bicontinuous cubic phase: The gyroid (la3d) forms at low hydration and the diamond (Pn3m) forms at high hydration (Spicer et al., 2002a). Identification of mesophases is predominantly performed using SAXS in combination with cryo-TEM imaging (Hyde, 2001; Rizwan et al., 2007). Therefore, SAXS was used in this study to investigate the bulk structural morphology of powders as well as of each dispersion prepared (Figure 2). Hydrated particles (Figure 2a) that did not contain OVA (blank and Quil-A containing particles) resulted in SAXS patterns characteristic of vesicles, whereas hydrated particles formulated with OVA showed SAXS patterns characteristic of the cubic $(\mathrm{Pn} 3 \mathrm{~m})$ phase (Bragg peaks at relative positions of $\sqrt{2}: \sqrt{3}: \sqrt{4}: \sqrt{6}: \sqrt{8}: \sqrt{9}$ ). Cubosomes with OVA and Quil-A and cubosomes with OVA alone had lattice parameters of $98 \AA$ and $95 \AA$, respectively. Interestingly, all dry powders resulted in SAXS patterns without peaks which is characteristic of vesicles (Figure $2 \mathrm{~b}$ ). This showed that cubosomes are not formed in the spray drying feed or during the spray drying process, but are formed upon rehydration of the powder. In a previous study where a liposome-based vaccine was manufactured and then spray dried, the authors reported that optimization of both formulation and processing conditions was necessary to stabilize the liposomes through the drying process (Ingvarsson et al., 2013). The method presented here skips the particle preparation step prior to spray drying and circumvents the need for such optimization since the cubosomes are only formed after rehydration of the powder. It thereby offers improved robustness and reduced costs.

Quil-A is a highly surface active compound which interacts with lipid membranes and disrupts these at high Quil-A concentrations (Demana et al., 2004). However, it has been reported not to disrupt liposomes at $2 \% \mathrm{w} / \mathrm{w}$ (Quil-A/lipid) (White et al., 2006). For this reason, Quil-A was used at $2 \% \mathrm{w} / \mathrm{w}$ (Quil-A/lipid) in this study and it was found that the addition of Quil-A did not change the morphology of the vesicles and cubosomes in the absence and presence of OVA, respectively. The slightly greater lattice parameter in the presence of Quil-A could be understood as a slight swelling due to the charge carried by Quil-A as observed for other ionic surfactants (Liu et al., 2013).

The structural morphology of hydrated OVA and Quil-A cubosomes was verified by cryo-TEM (Figure 3) that showed substructures characteristic of cubosomes (Hyde, 2001). The substructure of blank cubosomes was also investigated by cryo-TEM and while cubosomes were found, they were less abundant than in the OVA and Quil-A containing samples and instead more vesicles were seen. This could indicate that the lack of Pn3m SAXS signal seen from blank cubosomes was a consequence of a too low cubosome concentration caused by much of the lipid forming vesicles instead of cubosomes. These data thereby suggest that OVA has a stabilizing effect on cubosomes. It is yet to be seen if clinically relevant antigens will also provide this effect. However, in a case where it cannot, other polymeric stabilizers such as Poloxamer 407 may be added to achieve a similar effect (Spicer et al., 2001). 
a)

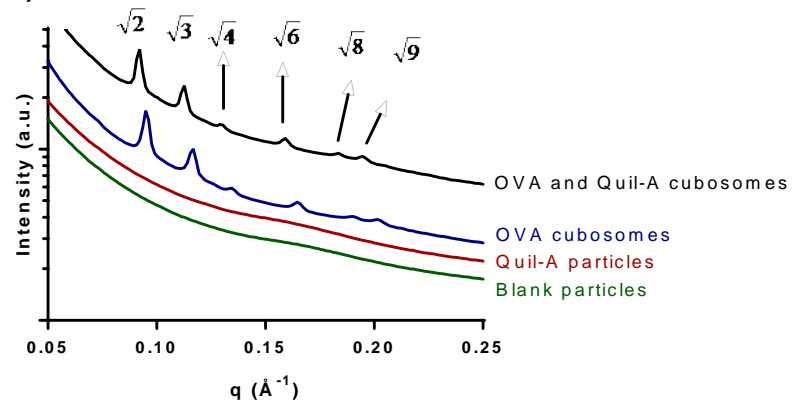

b)

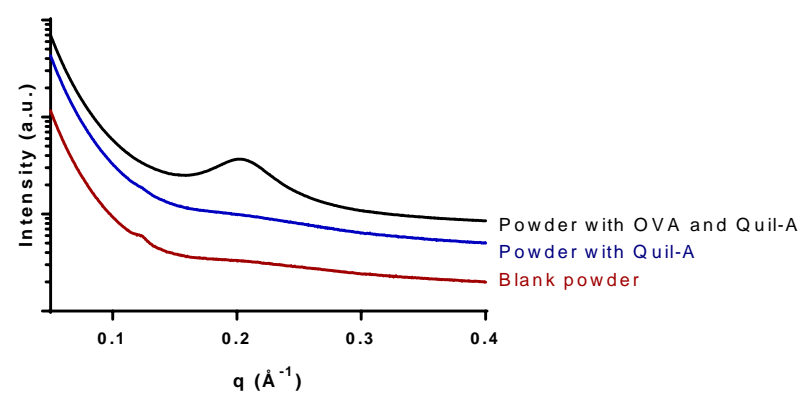

Figure 2. q vs intensity patterns obtained from SAXS measurements of a) dispersions in water of cubosomes with no Quil-A or OVA (blank), only Quil-A, only OVA, and OVA and Quil-A. b) Spray dried powders of monoolein and dextran with no Quil-A or OVA (blank), only Quil-A, and with OVA and Quil-A. Bragg peaks at relative positions of $\sqrt{2}: \sqrt{3}: \sqrt{4}: \sqrt{6}: \sqrt{8}: \sqrt{9}$ were seen in hydrated particles formulated with OVA as well as OVA and Quil-A and are marked on the figure.

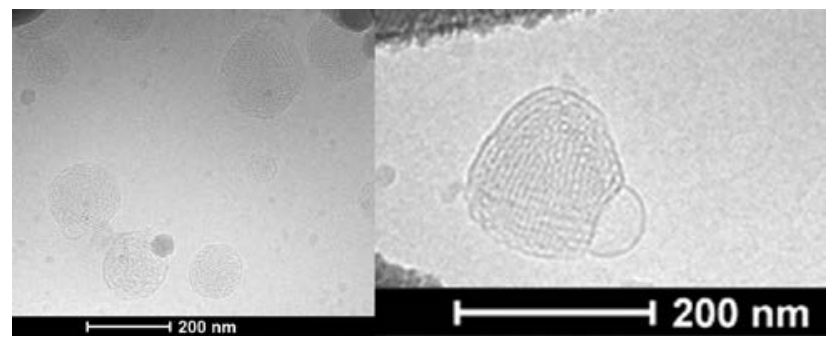

Figure 3. Representative cryo-TEM images of hydrated cubosomes containing OVA and Quil-A. The particles show substructures characteristic of cubosomes.

\section{Size, zeta potential and stability of cubosomes}

The size and surface properties of particulate vaccines affect their function, as reviewed by Bachmann et al. (Bachmann and Jennings, 2010). Cubosomes containing OVA and/or Quil-A as well as blanks were characterized for their size and polydispersity index (PDI) using DLS. All hydrated particles had average sizes in the nano-range with high homogeneity (PDI values between 0.1-0.2) (Table 2). OVA cubosomes had an average size of $316 \pm 21 \mathrm{~nm}$, whereas blank particles had a size of $134 \pm 7 \mathrm{~nm}$. The addition of Quil-A to blank particles (Quil-A particles) led to a small but significant $(p=0.0289)$ decrease in size to $120 \pm 3 \mathrm{~nm}$. However, the addition of Quil-A to OVA cubosomes (OVA and Quil-A cubosomes) led to a more pronounced and significant $(p=0.0104)$ decrease in size to $257 \pm 8 \mathrm{~nm}$. The presence of Quil-A in particles additionally led to a reduction of the PDI whether or not OVA was co-formulated. Antigen presenting cells have been reported in the literature to process particulates in the size range of $20 \mathrm{~nm}$ to $3 \mu \mathrm{m}$ and there is no clear evidence of an ideal size within this range (Bachmann and Jennings, 2010). All particles were hence of a size suited for vaccination. 
The zeta potential of particles is a measure of their surface charge and is often used as an indicator of the colloidal stability of the dispersion (Heurtault et al., 2010; Kallerup et al., 2017). It is reported in the literature that as a rule of thumb, particles with zeta potential of magnitude greater than $30 \mathrm{mV}$ will be colloidally stable and that a zeta potential of approximately $20 \mathrm{mV}$ is indicative of short-term colloidal stability. Particles with zeta potential of -5 to $5 \mathrm{mV}$ may be expected to agglomerate quickly (Honary and Zahir, 2013). In this study, the zeta potentials of all particles were negative. The zeta potential of blank particles was measured to be $-43.6 \pm 0.4 \mathrm{mV}$. However, addition of Quil-A and/or OVA reduced the zeta potential to approximately $-20 \mathrm{mV}$ indicating that the latter formulations will show limited colloidal stability. This was investigated further for OVA and Quil-A cubosomes where a dispersion was left to stand for $24 \mathrm{~h}$ without agitation and the average particle size was measured at various time points. The particle size and PDI gradually increased, indicating that the cubosomes were slowly agglomerating (Table 3 ). The difference was significant after $30 \mathrm{~min}(\mathrm{p}=0.0176)$, where the particle size had increased to $299 \pm 11 \mathrm{~nm}$, and after $24 \mathrm{~h}$ the size was $451 \pm 12 \mathrm{~nm}$. However, as discussed above, this increased size is not likely to be of importance for the immune response to the formulation. The morphological stability of cubosomes with OVA and Quil-A was also investigated (Figure 4) and it was found that the structure of the cubosomes was unchanged for at least $26 \mathrm{~h}$ in an aqueous dispersion. Thus, the agglomeration of the cubosomes seems not to affect the particle morphology.

Table 2. Size, PDI and zeta potential of particles dispersed in $9.5 \mathrm{mM}$ PBS at pH 7.3 (size and PDI) or phosphate buffer at $\mathrm{pH} 7.3$ without added salts (zeta potential) measured by DLS. Data represents mean \pm $\operatorname{SD}(n=3)$.

\begin{tabular}{|l|l|l|l|}
\hline Formulation & size $(\mathbf{n m})$ & PDI & Zeta potential $(\mathbf{m V})$ \\
\hline Blank particles & $134 \pm 7$ & $0.19 \pm 0.04$ & $-43.6 \pm 0.4$ \\
\hline Particles with Quil-A & $120 \pm 3$ & $0.11 \pm 0.00$ & $-17.0 \pm 1.0$ \\
\hline Cubosomes with OVA & $316 \pm 21$ & $0.23 \pm 0.02$ & $-20.9 \pm 0.4$ \\
\hline Cubosomes with OVA and Quil-A & $257 \pm 8$ & $0.11 \pm 0.08$ & $-18.0 \pm 0.6$ \\
\hline
\end{tabular}

Table 3. Colloidal stability in $9.5 \mathrm{mM}$ PBS at $\mathrm{pH} 7.3$ for OVA and Quil-A cubosomes measured over $24 \mathrm{~h}$ by DLS. Data represents mean $\pm S D(n=3)$.

\begin{tabular}{|l|l|l|}
\hline Time & size $(\mathrm{nm})$ & PDI \\
\hline $0 \mathrm{~h}$ & $264 \pm 11$ & $0.15 \pm 0.03$ \\
\hline $0.5 \mathrm{~h}$ & $299 \pm 11$ & $0.21 \pm 0.02$ \\
\hline $6 \mathrm{~h}$ & $354 \pm 12$ & $0.26 \pm 0.01$ \\
\hline
\end{tabular}




\begin{tabular}{|l|l|l|}
\hline $24 \mathrm{~h}$ & $451 \pm 12$ & $0.31 \pm 0.03$ \\
\hline
\end{tabular}

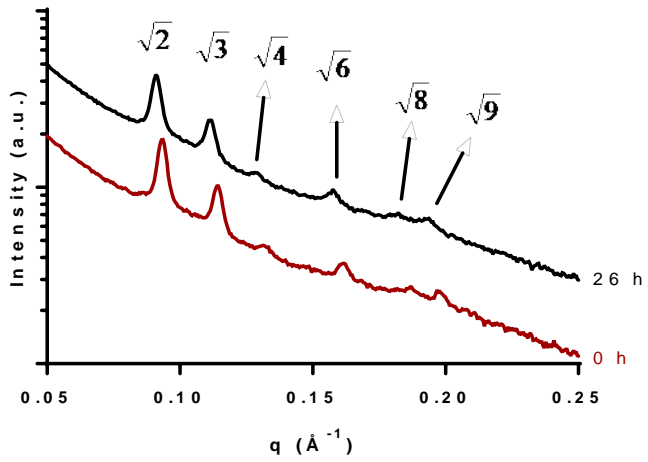

Figure 4. $q$ vs intensity patterns obtained from SAXS measurements of a dispersion of cubosomes with OVA and Quil-A in PBS pH 7.3 at $37^{\circ} \mathrm{C}$ at 0 and $26 \mathrm{~h}$ after reconstitution. Bragg peaks at relative positions of $\sqrt{2}: \sqrt{3}: \sqrt{4}: \sqrt{6}: \sqrt{8}: \sqrt{9}$ were seen at both time points and are marked on the figure.

\section{OVA loading, encapsulation efficiency and release of OVA from cubosomes}

To quantitate the OVA load and release, FITC-labeled OVA was used. The total FITC-OVA content in the spray dried powder of OVA and Quil-A cubosome precursors was measured after dissolving the cubosomes. The content was found to be $106.0 \pm 0.7 \mu \mathrm{g} / \mathrm{mg}$ of FITC-OVA in the bulk powder corresponding to the composition of the liquid mixture before spray drying. Vaccines are generally administered by injection s.c. or intra-muscularly. However, due to the multitude of benefits offered by oral administration over injection, there have been many efforts to allow oral administration of drugs and vaccines (Sahni et al., 2016). A promising strategy is to pack the vaccine into advanced delivery systems such as microcontainers (Amenitsch et al., 1998; Nielsen et al., 2017). The high OVA content in the cubosomes presented here makes this formulation well suited for coupling with such administration systems.

The release of FITC-OVA from the OVA and Quil-A cubosomes was measured over $48 \mathrm{~h}$ in $9.5 \mathrm{mM} \mathrm{PBS}, \mathrm{pH}$ 7.3 (Figure 5). Immediately after reconstitution, $65 \%$ of the loaded OVA was free in solution and then a gradual release was observed until all FITC-OVA was released at $24 \mathrm{~h}$. Entrapment efficiency of FITC-OVA in the cubosomes was evaluated as the relative amount of FITC-OVA not released during rehydration (a 20 min process) of the cubosomes and was determined to be $35 \pm 1 \%$ (mean $\pm S D, n=4$ ). The $65 \%$ FITC-OVA measured outside the cubosomes after reconstitution is in contrast to a similar cubosome formulation with OVA, where a limited burst release upon hydration of the cubosome precursors was reported (Nielsen et al., 2017). Nielsen et al. speculated that the observed burst release was actually not a burst release from the particles, but instead dissolution of free OVA in the powder (Nielsen et al., 2017). This notion is supported by the lack of a burst release in another study where the cubosomes were not formulated as powder, but instead directly as a concentrated suspension of cubosomes (Rizwan et al., 2013). The study here and the one presented in the literature (Nielsen et al., 2017) used similar methods to produce the 
formulations, but in this study a significantly higher OVA concentration was added to the cubosomes. Hence, the larger burst release seen in this study is likely to be related to a relatively larger amount of FITCOVA present outside the cubosomes in the powder rather than entrapped in or associated to the cubosome precursors. Another difference to the work by Nielsen et al. is the addition of Quil-A to the cubosome precursors in the current study. Quil-A has previously been reported to reduce the OVA-peptide entrapment of cubosomes by a factor of three (Rattanapak et al., 2012) and a similar effect of the addition of Quil-A could be speculated to be the case in this study. The addition of Quil-A may also be the explanation for the increased release rate of FITC-OVA seen in this study compared to the very slow and incomplete release after 4-10 days reported in earlier studies (Nielsen et al., 2017; Rizwan et al., 2013).

Physical linkage of antigen to adjuvant, either by direct interaction of antigen and adjuvant or by entrapment, encapsulation or adsorption of antigen and adjuvant to a particle (e.g. liposomes), has been widely reported to be important for the immunogenicity of a vaccine as reviewed by Kamath et al (Kamath et al., 2012). However, White et al. reported similar levels of antigen specific cytotoxicity after vaccination with Quil-A containing liposomes admixed with free antigen as with liposomes containing both antigen and Quil-A (White et al., 2006). Physically linking the antigen to the particle/adjuvant or delivery of soluble antigen together with particle/adjuvant will therefore likely cause a similar immune response.

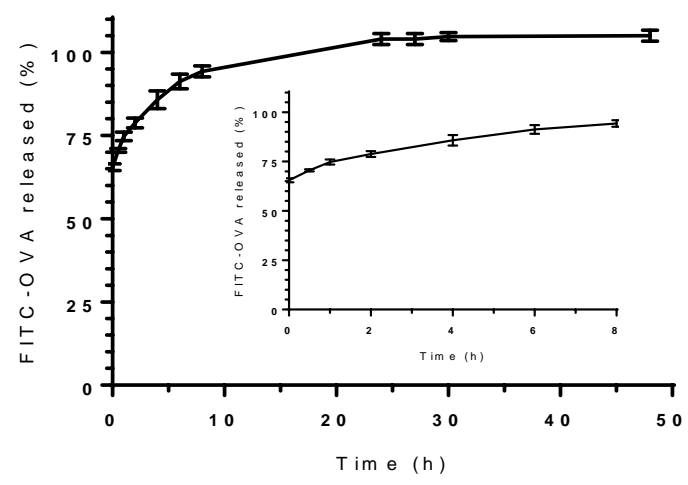

Figure 5. Release of FITC-OVA from cubosomes (also containing Quil-A) in $9.5 \mathrm{mM} \mathrm{PBS}$ at pH 7.3 and $37^{\circ} \mathrm{C}$. The insert shows the release over the first $8 \mathrm{~h}$. Data are expressed as \% released of total OVA content and are represented as mean \pm SD $(n=4)$. The aliquots used for the $0 \mathrm{~h}$ time-point were taken immediately after a 20 min reconstitution process.

\section{Stability of secondary structure of OVA during formulation and storage}

$C D$ of hydrated cubosomes was used to verify preservation of the secondary structure of OVA during production and storage. Native OVA and OVA boiled for $2 \mathrm{~h}$ were used as controls for preserved and changed secondary structure respectively. The CD spectra from both controls were similar to those reported in the literature (Hamborg et al., 2013). All cubosome samples showed similar CD patterns to the control of native OVA (representative spectra are shown in Figure 6, all spectra are shown in Figure S2) 
indicating a preserved secondary structure of OVA after both spray drying and the secondary drying step $\left(86^{\circ} \mathrm{C}\right.$ for $\left.24 \mathrm{~h}\right)$, as well as after storage for 6 months at room temperature under dry conditions. Lyophilized OVA was also exposed to the secondary drying step and a preserved secondary structure was observed (Figure S3) indicating that dry OVA was stable. Preservation of the secondary structure of OVA during spray drying of cubosomes may thus be due to the high stability of OVA, but may also result from spray drying being a low temperature method with a fast drying step. In the early part of the spray drying process, the drying air is hot but the surface of the particles is wet and thus evaporation will cause constant loss of heat keeping the droplet temperature much lower than the drying gas temperature. This effect will eventually disappear, but at this stage the labile compounds will be dry and the drying gas will have cooled due to the preceding heat-loss due to evaporation (Ameri and Maa, 2006). Since OVA on its own is stable during the secondary drying step, one may consider whether clinically relevant peptides may be damaged by similar treatments. However, the secondary drying step was merely implemented for accurate in vitro characterization of the formulations and may be omitted if desired. Additionally, it is worth noting that denatured forms of proteins in some cases stimulate stronger immune responses than native forms, especially in the case of cell mediated immune responses, and therefore heat-denaturation of the antigen might not be undesirable (Speidel et al., 1997).

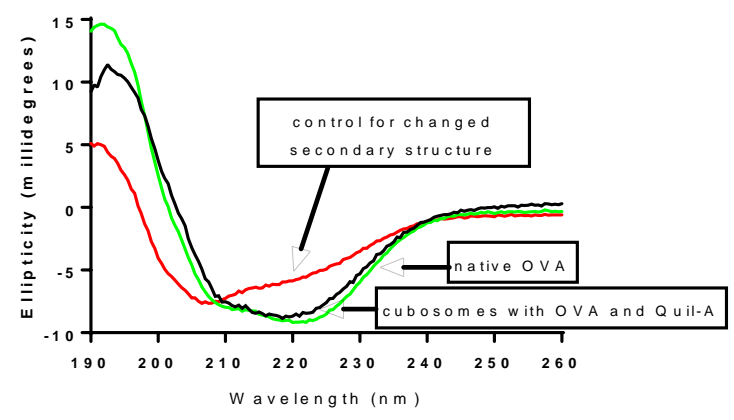

Figure 6. Representative CD spectra of OVA and Quil-A containing cubosomes after spray drying and secondary drying at $86^{\circ} \mathrm{C}$ for $24 \mathrm{~h}$ (solid black). The CD spectrum of a control for preserved secondary structure (native OVA) is shown in green and for changed secondary structure (OVA boiled for $2 \mathrm{~h}$ ) is shown in red. The concentration of OVA was $106 \mu \mathrm{g} / \mathrm{mL}$ in all measurements.

\section{Serum IgG levels in vaccinated mice}

Antigen specific serum IgG is often used as an indicator of systemic humoral immune reactivity. Mice were immunized with cubosomes with OVA and Quil-A, cubosomes without antigen (negative control), unformulated OVA and Quil-A, and OVA in alum (positive control) and anti-OVA serum IgG was measured (Figure 7). Alum has been used widely as a vaccine adjuvant and is known to be a strong stimulator of antibody responses (Oleszycka and Lavelle, 2014). The use of Quil-A in human vaccines has so far been limited due to the occurrence of injection site reactions thus making it desirable to use a low dose (Hook and Rades, 2013). The cubosomes prepared in this study contained Quil-A as adjuvant at a 1:15 (w/w) ratio to OVA giving a low parenteral Quil-A dose of $0.67 \mu \mathrm{g}$ (Myschik et al., 2008b). Nonetheless, OVA and Quil-A 
in cubosomes elicited significantly more total antigen-specific serum $\lg G$ than alum $(p<0.01)$ and all other controls $(p<0.001)$, showing that they induce a strong humoral immune response. This dry powder cubosome formulation appeared to be equally as effective as cubosomes produced from solution with monophosphoryl lipid A (MPL) and imiquimod as adjuvants (Rizwan et al., 2013). However, OVA and Quil-A given orally either in PBS or in cubosomes did not elicit an antibody response, thus indicating that a better delivery system is needed for oral delivery of cubosomes.

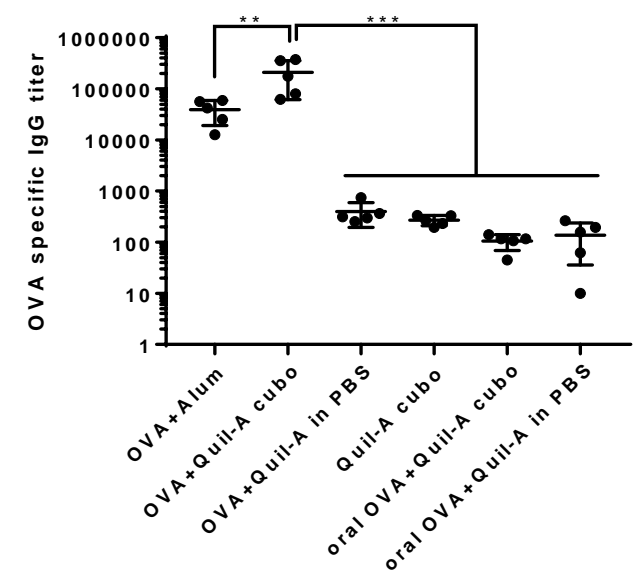

Figure 7. OVA specific serum IgG antibody titers. The data are from individual mice from a representative experiment of three independent experiments together with mean \pm standard deviation $(n=5$ mice/experiment). ${ }^{* *} \mathrm{p}<0.01,{ }^{* * *} \mathrm{p}<0.001$.

\section{Antigen-specific cell expansion assay and in vivo cytotoxicity assay}

Mice received antigen-specific transgenic $\mathrm{CD}^{+}$and $\mathrm{CD}^{+}$cells from OT-I and OT-II mice by adoptive transfer prior to vaccination. OT-I mice have been genetically engineered to express a high proportion of $\mathrm{CD}^{+} \mathrm{T}$ cells with a $\mathrm{T}$ cell receptor specific for the OVA $\mathrm{A}_{257-264}$ peptide and OT-II mice to express $\mathrm{CD} 4^{+} \mathrm{T}$ cells with a $T$ cell receptor specific for the $\mathrm{OVA}_{323-339}$ peptide (Kojarunchitt et al., 2015). To investigate the cellular immune response to the cubosomes, expansion of antigen-specific $T$ cells $\left(v_{\alpha} 2^{+} v_{\beta} 5^{+}\right)$was assessed 6 days after the last immunization (Figure 8 ). A significantly greater expansion of $C D 8^{+}$cells was observed in spleens and lymph nodes from mice immunized s.c. with cubosomes with OVA and Quil-A compared to all controls ( $p<0.001$, Figure $8 a$ and Figure $8 b$ ). The response to unformulated OVA and Quil-A was comparable to that to alum, which is known to be a weak adjuvant for stimulating a cellular response (Oleszycka and Lavelle, 2014). This indicates that s.c. administration of cubosomes with Quil-A can stimulate a strong cytotoxic $\mathrm{T}$ cell immune response important for protection against cytosolic infections. Orally administered cubosomes had no effect.

$\mathrm{CD}^{+} \mathrm{T}$ cell responses are important for activating, improving and controlling immune responses and thus an ideal vaccine will elicit a CD4 ${ }^{+} \mathrm{T}$ cell response (Rosa et al., 2010). Cubosomes with OVA and Quil-A could induce antigen specific $\mathrm{CD} 4^{+}$expansion comparable to alum in both spleens and lymph nodes (Figure $8 \mathrm{c}$ and Figure $8 d)$. The response was significantly stronger than control without antigen $(p<0.01)$ but comparable 
to unformulated OVA and Quil-A. A small $\mathrm{CD}^{+}$response was observed from oral unformulated OVA and Quil-A, but no response was seen from oral cubosomes.

a)

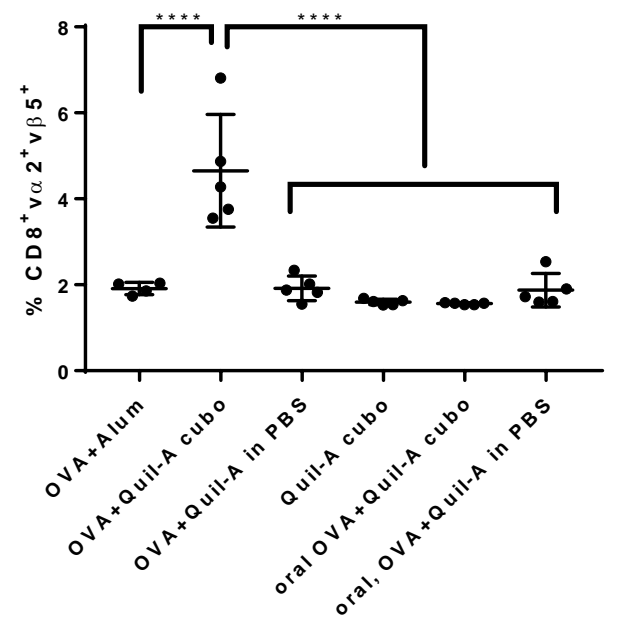

c)

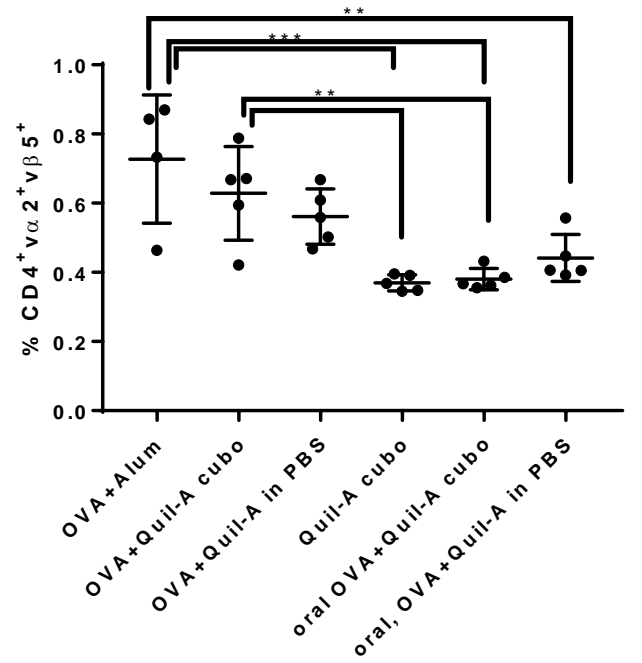

b)

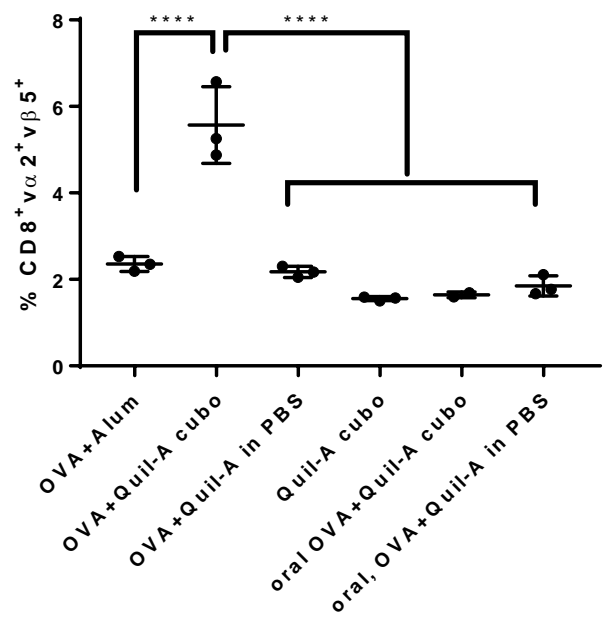

d)

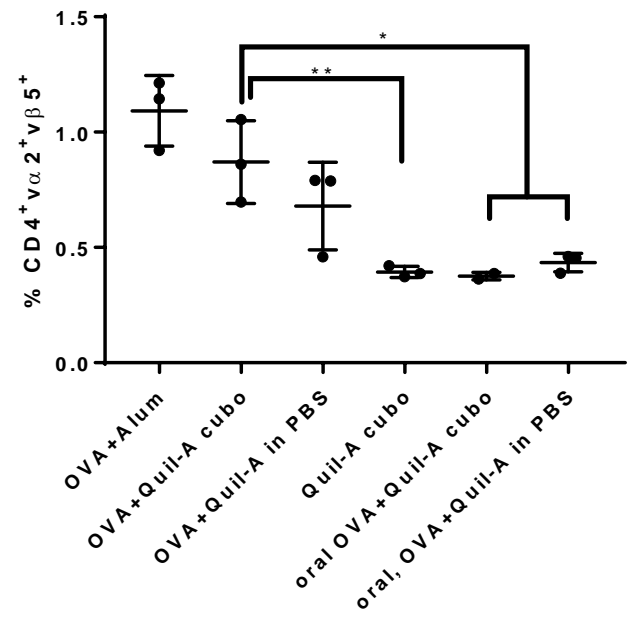

Figure 8. In vivo expansion of transgenic $C D 8^{+}$cells ( $a$ and $b$ ) and $C D 4^{+}$cells ( $c$ and $d$ ) in spleens ( $a$ and $c$ ) and lymph nodes ( $b$ and $d)$. Data in a and $c$ are results from individual mice plus the mean and SD from a representative experiment of three independent experiments ( $n=5$ mice/experiment). Data in $b$ and $d$ are pooled data from 4-5 mice in each of three independent experiments. ${ }^{*} p<0.05,{ }^{* *} p<0.01,{ }^{* * *} p<0.001$, $* * * * \mathrm{p}<0.0001$.

The antigen specific cytotoxic T cell response was further investigated through an in vivo cytotoxicity assay by measuring the killing of OVA-peptide pulsed cells relative to unpulsed cells (Figure 9). Quil-A has 
received great interest for its ability to stimulate $\mathrm{CD} 8^{+}$cytotoxic $\mathrm{T}$ cell responses (Hook and Rades, 2013). In this study, both OVA with alum and OVA in solution with Quil-A resulted in significantly greater target cell death than the control without OVA $(p<0.0001)$. In agreement with the cell expansion data, mice immunized with OVA and Quil-A in cubosomes provided the highest level of killing of OVA-labelled target cells, with almost total removal of target cells causing saturation of the assay. The assay saturation is likely the reason why the cubosomes did not cause significantly more killing than unformulated OVA and Quil-A or OVA with alum. When administered orally, unformulated OVA and Quil-A potentially had some effect, but this was not significant. Orally administered cubosomes with OVA and Quil-A had no effect which is an agreement with the transgenic cell expansion results.

In summary, s.c. administered cubosomes stimulated both humoral and cellular immune responses to OVA and this was not seen after oral administration. It is possible that an effect could have been obtained using a more advanced oral vaccine delivery system (Davitt and Lavelle, 2015; McHugh et al., 2017). One such system is microcontainers presented in the literature (Chirra et al., 2014; Mazzoni et al., 2017; Nielsen et al., 2016). These offer protection of their load through the stomach; however, they have limited internal volume and therefore limited loading capacity for the formulations they deliver. The stability at room temperature and high antigen load of the cubosome powder presented here renders it well suited for future application with microcontainers.

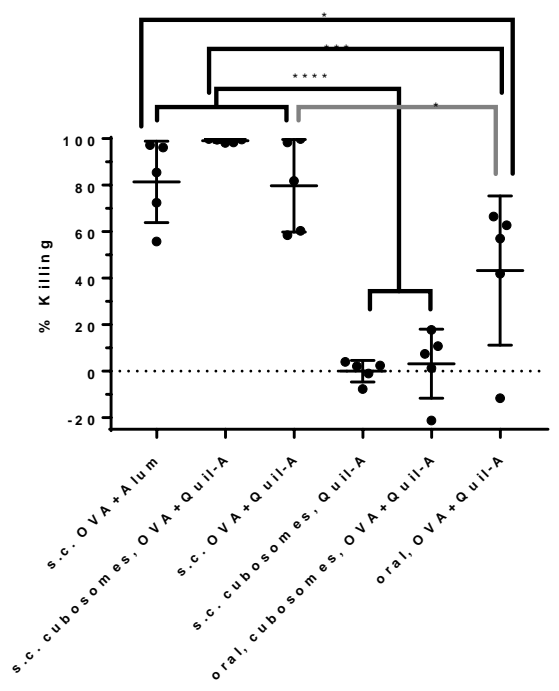

Figure 9. In vivo killing of adoptively transferred OVA-peptide labelled T cells in spleens. Data shown are results from individual mice plus the mean and SD from a representative experiment of three independent experiments ( $\mathrm{n}=5$ mice/experiment). ${ }^{*} \mathrm{p}<0.05,{ }^{* * *} \mathrm{p}<0.001,{ }^{* * * *} \mathrm{p}<0.0001$.

\section{Conclusion}

Dry powder precursors of cubosomes containing the model antigen ovalbumin and the adjuvant Quil-A were produced in a simple one-step spray drying process. The powder precursors were stable at room 
temperature and formed cubosomes in the nanometer-size range upon hydration. These cubosomes provided effective vaccination when administrated s.c. in mice but no effect was observed after oral administration. Since this vaccine is in a powder form with a high antigen load, it is well suited for application with microfabricated administration systems such as microcontainers.

\section{Acknowledgements}

This work was supported by the Danish National Research Foundation (DNRF122) and Villum Fonden (Grant No. 9301) for Intelligent Drug Delivery and Sensing Using Microcontainers and Nanomechanics (IDUN). Line Hagner Nielsen would like to thank the Danish Research Council for Technology and Production (FTP), Project DFF 4004-00120B for financial support.

The authors would like to acknowledge Nanna Bild, Technical University of Denmark, for the drawing of the schematic, and the Core Facility for Integrated Microscopy, Faculty of Health and Medical Sciences, University of Copenhagen is thanked for the cryo-TEM studies. Ingrid Elise Weidahl is acknowledged for work on optimization of the spray drying process. The scattering experiments in this manuscript were performed on the SAXS/WAXS beamline at the Australian Synchrotron, Clayton, Australia and at the Austrian SAXS/WAXS beamline at the synchrotron light source ELETTRA (Trieste, Italy). The authors are grateful to Kan Kaneko for his help establishing the in vivo protocols.

\section{Conflicts of interest}

The authors have no conflicts of interest to declare.

\section{Supporting information}

Gating strategy for FACS data

Circular dichroism figures with measurement of both batches of cubosomes with OVA and Quil-A and all controls. 


\section{Bibliography}

Ali, M.A., Kataoka, N., Ranneh, A.-H., Iwao, Y., Noguchi, S., Oka, T., Itai, S., 2017. Enhancing the Solubility and Oral Bioavailability of Poorly Water-Soluble Drugs Using Monoolein Cubosomes. Chem. Pharm. Bull. (Tokyo). 65, 42-48. https://doi.org/10.1248/cpb.c16-00513

Amenitsch, H., Rappolt, M., Kriechbaum, M., Mio, H., Laggner, P., Bernstorff, S., 1998. First performance assessment of the small-angle X-ray scattering beamline at ELETTRA. J. Synchrotron Radiat. 5, 506508. https://doi.org/10.1107/S090904959800137X

Ameri, M., Maa, Y.F., 2006. Spray drying of biopharmaceuticals: Stability and process considerations. Dry. Technol. 24, 763-768. https://doi.org/10.1080/03602550600685275

Avachat, A.M., Parpani, S.S., 2015. Formulation and development of bicontinuous nanostructured liquid crystalline particles of efavirenz. Colloids Surfaces B Biointerfaces 126, 87-97. https://doi.org/10.1016/j.colsurfb.2014.12.014

Azizi, A., Kumar, A., Diaz-Mitoma, F., Mestecky, J., 2010. Enhancing Oral Vaccine Potency by Targeting Intestinal M Cells. PLoS Pathog. 6, e1001147. https://doi.org/10.1371/journal.ppat.1001147

Azmi, F., Ahmad Fuaad, A.A.H., Skwarczynski, M., Toth, I., 2014. Recent progress in adjuvant discovery for peptide-based subunit vaccines. Hum. Vaccin. Immunother. 10, 778-796. https://doi.org/10.4161/hv.27332

Bachmann, M.F., Jennings, G.T., 2010. Vaccine delivery: A matter of size, geometry, kinetics and molecular patterns. Nat. Rev. Immunol. 10, 787-796. https://doi.org/10.1038/nri2868

Bei, D., Zhang, T., Murowchick, J.B., Youan, B.-B.C., 2010. Formulation of Dacarbazine-loaded Cubosomes. Part III. Physicochemical Characterization. AAPS PharmSciTech 11, 1243-1249. https://doi.org/10.1208/s12249-010-9496-7

Caltagirone, C., Falchi, A.M., Lampis, S., Lippolis, V., Meli, V., Monduzzi, M., Prodi, L., Schmidt, J., Sgarzi, M., Talmon, Y., Bizzarri, R., Murgia, S., 2014. Cancer-cell-targeted theranostic cubosomes. Langmuir 30, 6228-6236. https://doi.org/10.1021/la501332u

Chirra, H.D., Shao, L., Ciaccio, N., Fox, C.B., Wade, J.M., Ma, A., Desai, T.A., 2014. Planar Microdevices for Enhanced In Vivo Retention and Oral Bioavailability of Poorly Permeable Drugs. Adv. Healthc. Mater. 3, 1648-1654. https://doi.org/10.1002/adhm.201300676

Davitt, C.J.H., Lavelle, E.C., 2015. Delivery strategies to enhance oral vaccination against enteric infections. Adv. Drug Deliv. Rev. 91, 52-69. https://doi.org/10.1016/j.addr.2015.03.007

Demana, P.H., Fehske, C., White, K., Rades, T., Hook, S., 2004. Effect of incorporation of the adjuvant Quil A on structure and immune stimulatory capacity of liposomes. Immunol. Cell Biol. 82, 547-554. https://doi.org/10.1111/j.0818-9641.2004.01276.x

Ehreth, J., 2003. The value of vaccination: a global perspective. Vaccine 21, 4105-4117. https://doi.org/10.1016/S0264-410X(03)00377-3 
Fang, Z., Bhandari, B., 2017. Spray Drying of Bioactives, in: Engineering Foods for Bioactives Stability and Delivery. pp. 261-284. https://doi.org/10.1007/978-1-4939-6595-3_10

Foged, C., 2011. Subunit vaccines of the future: the need for safe, customized and optimized particulate delivery systems. Ther. Deliv. 2, 1057-1077. https://doi.org/10.4155/tde.11.68

Gebril, A., Alsaadi, M., Acevedo, R., Mullen, A.B., Ferro, V.A., 2012. Optimizing efficacy of mucosal vaccines. Expert Rev. Vaccines 11, 1139-1155. https://doi.org/10.1586/erv.12.81

Gordon, S., Young, K., Wilson, R., Rizwan, S., Kemp, R., Rades, T., Hook, S., 2012. Chitosan hydrogels containing liposomes and cubosomes as particulate sustained release vaccine delivery systems. J. Liposome Res. 22, 193-204. https://doi.org/10.3109/08982104.2011.637502

Hamborg, M., Jorgensen, L., Bojsen, A.R., Christensen, D., Foged, C., 2013. Protein antigen adsorption to the DDA/TDB liposomal adjuvant: effect on protein structure, stability, and liposome physicochemical characteristics. Pharm. Res. 30, 140-55. https://doi.org/10.1007/s11095-012-0856-8

Heeg, K., Kuon, W., Wagner, H., 1991. Vaccination of class I major histocompatibility complex (MHC)restricted murine CD8+ cytotoxic $\mathrm{T}$ lymphocytes towards soluble antigens: immunostimulatingovalbumin complexes enter the class I MHC-restricted antigen pathway and allow sensitization against t. Eur. J. Immunol. 21, 1523-1527. https://doi.org/10.1002/eji.1830210628

Heurtault, B., Frisch, B., Pons, F., 2010. Liposomes as delivery systems for nasal vaccination: strategies and outcomes. Expert Opin. Drug Deliv. 7, 829-44. https://doi.org/10.1517/17425247.2010.488687

Hill, A.B., Kilgore, C., McGlynn, M., Jones, C.H., 2016. Improving global vaccine accessibility. Curr. Opin. Biotechnol. 42, 67-73. https://doi.org/10.1016/j.copbio.2016.03.002

Honary, S., Zahir, F., 2013. Effect of Zeta Potential on the Properties of Nano - Drug Delivery Systems - A Review (Part 2). Trop. J. Pharm. Res. 12, 265-273. https://doi.org/10.4314/tjpr.v12i2.19

Hook, S., Rades, T., 2013. Immune stimulating complexes (ISCOMs) and Quil-A containing particulate formulations as vaccine delivery systems, in: Flower, D.R., Perrie, Y. (Eds.), Immunomic Discovery of Adjuvants and Candidate Subunit Vaccines. Immunomics Reviews: (An Official Publication of the International Immunomics Society). Springer, New York, pp. 233-261. https://doi.org/10.1007/978-14614-5070-2_12

Hyde, S.T., 2001. Identification of lyotropic liquid crystal mesophases, in: Holmberg, K. (Ed.), Handbook of Applied Surface and Colloid Chemistry. John Wiley \& Sons, Ltd., New York, pp. 299-332.

Ingvarsson, P.T., Schmidt, S.T., Christensen, D., Larsen, N.B., Hinrichs, W.L.J., Andersen, P., Rantanen, J., Nielsen, H.M., Yang, M., Foged, C., 2013. Designing CAF-adjuvanted dry powder vaccines: spray drying preserves the adjuvant activity of CAF01. J. Control. Release 167, 256-64. https://doi.org/10.1016/j.jconrel.2013.01.031

Kallerup, R.S., Franzyk, H., Schiøth, M.L., Justesen, S., Martin-Bertelsen, B., Rose, F., Madsen, C.M., Christensen, D., Korsholm, K.S., Yaghmur, A., Foged, C., 2017. Adjuvants Based on Synthetic 
Mycobacterial Cord Factor Analogues: Biophysical Properties of Neat Glycolipids and NanoselfAssemblies with DDA. Mol. Pharm. 14, 2294-2306. https://doi.org/10.1021/acs.molpharmaceut.7b00170

Kamath, A.T., Mastelic, B., Christensen, D., Rochat, A.-F., Agger, E.M., Pinschewer, D.D., Andersen, P., Lambert, P.-H., Siegrist, C.-A., 2012. Synchronization of dendritic cell activation and antigen exposure is required for the induction of Th1/Th17 responses. J. Immunol. 188, 4828-37. https://doi.org/10.4049/jimmunol.1103183

Kensil, C.R., Wu, J.-Y., Soltysik, S., 1995. Structural and Immunological Characterization of the of the Vaccine Adjuvant QS-21. Pharm. Biotechnol. 6, 525-41. https://doi.org/10.1007/978-1-4615-1823-5_22

Kersten, G.F.A., Crommelin, D.J.A., 2003. Liposomes and ISCOMs. Vaccine 21, 915-920. https://doi.org/10.1016/S0264-410X(02)00540-6

Kirby, N.M., Mudie, S.T., Hawley, A.M., Cookson, D.J., Mertens, H.D.T., Cowieson, N., Samardzic-Boban, V., 2013. A low-background-intensity focusing small-angle X-ray scattering undulator beamline. J. Appl. Crystallogr. 46, 1670-1680. https://doi.org/10.1107/S002188981302774X

Kojarunchitt, T., Baldursdottir, S., Dong, Y. Da, Boyd, B.J., Rades, T., Hook, S., 2015. Modified thermoresponsive Poloxamer 407 and chitosan sol-gels as potential sustained-release vaccine delivery systems. Eur. J. Pharm. Biopharm. 89, 74-81. https://doi.org/10.1016/j.ejpb.2014.11.026

Könnings, S., Copland, M.J., Davies, N.M., Rades, T., 2002. A method for the incorporation of ovalbumin into immune stimulating complexes prepared by the hydration method. Int. J. Pharm. 241, 385-389. https://doi.org/10.1016/S0378-5173(02)00270-3

Liu, Q., Dong, Y. Da, Hanley, T.L., Boyd, B.J., 2013. Sensitivity of nanostructure in charged cubosomes to phase changes triggered by ionic species in solution. Langmuir 29, 14265-14273. https://doi.org/10.1021/la402426y

Liu, Z., Luo, L., Zheng, S., Niu, Y., Bo, R., Huang, Y., Xing, J., Li, Z., Wang, D., 2016. Cubosome nanoparticles potentiate immune properties of immunostimulants. Int. J. Nanomedicine 11, 3571-83. https://doi.org/10.2147/IJN.S110406

Mazzoni, C., Tentor, F., Strindberg, S.A., Nielsen, L.H., Keller, S.S., Alstrøm, T.S., Gundlach, C., Müllertz, A., Marizza, P., Boisen, A., 2017. From concept to in vivo testing: Microcontainers for oral drug delivery. J. Control. Release 268, 343-351. https://doi.org/10.1016/j.jconrel.2017.10.013

McHugh, K.J., Nguyen, T.D., Linehan, A.R., Yang, D., Behrens, A.M., Rose, S., Tochka, Z.L., Tzeng, S.Y., Norman, J.J., Anselmo, A.C., Xu, X., Tomasic, S., Taylor, M.A., Lu, J., Guarecuco, R., Langer, R., Jaklenec, A., 2017. Fabrication of fillable microparticles and other complex 3D microstructures. Science (80-. ). 357, 1138-1142. https://doi.org/10.1126/science.aaf7447

Myschik, J., Mcburney, W.T., Hennessy, T., Phipps-Green, A., Rades, T., Hook, S., 2008a. Immunostimulatory biodegradable implants containing the adjuvant Quil-A-Part II: In vivo evaluation. J. Drug Target. 16, 224-232. https://doi.org/10.1080/10611860701848886 
Myschik, J., McBurney, W.T., Rades, T., Hook, S., 2008b. Immunostimulatory lipid implants containing QuilA and DC-cholesterol. Int. J. Pharm. 363, 91-98. https://doi.org/10.1016/j.ijpharm.2008.07.014

Nielsen, L.H., Melero, A., Keller, S.S., Jacobsen, J., Garrigues, T., Rades, T., Müllertz, A., Boisen, A., 2016. Polymeric microcontainers improve oral bioavailability of furosemide. Int. J. Pharm. 504, 98-109. https://doi.org/10.1016/j.ijpharm.2016.03.050

Nielsen, L.H., Rades, T., Boyd, B., Boisen, A., 2017. Microcontainers as an oral delivery system for spray dried cubosomes containing ovalbumin. Eur. J. Pharm. Biopharm. 118, 13-20. https://doi.org/10.1016/j.ejpb.2016.12.008

Nielsen, L.S., Schubert, L., Hansen, J., 1998. Bioadhesive drug delivery systems. I. Characterisation of mucoadhesive properties of systems based on glyceryl mono-oleate and glyceryl monolinoleate. Eur. J. Pharm. Sci. 6, 231-239. https://doi.org/10.1016/S0928-0987(97)10004-5

Ohtake, S., Martin, R.A., Yee, L., Chen, D., Kristensen, D.D., Lechuga-Ballesteros, D., Truong-Le, V., 2010. Heat-stable measles vaccine produced by spray drying. Vaccine 28, 1275-1284. https://doi.org/10.1016/j.vaccine.2009.11.024

Oleszycka, E., Lavelle, E.C., 2014. Immunomodulatory properties of the vaccine adjuvant alum. Curr. Opin. Immunol. 28, 1-5. https://doi.org/10.1016/j.coi.2013.12.007

Patton, J., Carey, M., 1979. Watching fat digestion. Science (80-. ). 204, 145-148. https://doi.org/10.1126/science.432636

Rattanapak, T., Young, K., Rades, T., Hook, S., 2012. Comparative study of liposomes, transfersomes, ethosomes and cubosomes for transcutaneous immunisation: characterisation and in vitro skin penetration. J. Pharm. Pharmacol. 64, 1560-9. https://doi.org/10.1111/j.2042-7158.2012.01535.x

Rizwan, S.B., Assmus, D., Boehnke, A., Hanley, T., Boyd, B.J., Rades, T., Hook, S., 2011. Preparation of phytantriol cubosomes by solvent precursor dilution for the delivery of protein vaccines. Eur. J. Pharm. Biopharm. 79, 15-22. https://doi.org/10.1016/j.ejpb.2010.12.034

Rizwan, S.B., Dong, Y.D., Boyd, B.J., Rades, T., Hook, S., 2007. Characterisation of bicontinuous cubic liquid crystalline systems of phytantriol and water using cryo field emission scanning electron microscopy (cryo FESEM). Micron 38, 478-485. https://doi.org/10.1016/j.micron.2006.08.003

Rizwan, S.B., McBurney, W.T., Young, K., Hanley, T., Boyd, B.J., Rades, T., Hook, S., 2013. Cubosomes containing the adjuvants imiquimod and monophosphoryl lipid A stimulate robust cellular and humoral immune responses. J. Control. Release 165, 16-21. https://doi.org/10.1016/j.jconrel.2012.10.020

Rosa, D.S., Ribeiro, S.P., Cunha-Neto, E., 2010. CD4+ T Cell Epitope Discovery and Rational Vaccine Design. Arch. Immunol. Ther. Exp. (Warsz). 58, 121-130. https://doi.org/10.1007/s00005-010-0067-0

Sahni, N., Cheng, Y., Russell Middaugh, C., Volkin, D.B., 2016. Vaccine Delivery: Current Routes of Administration and Novel Approaches, in: Drug Delivery: Principles and Applications: Second Edition. 
John Wiley \& Sons, Inc, Hoboken, NJ, pp. 623-654. https://doi.org/10.1002/9781118833322.ch24

Searles, J., Cherian, M., 2015. Alternatives to Vial Lyophilization, in: Lyophilized Biologics and Vaccines. Springer New York, New York, NY, pp. 257-271. https://doi.org/10.1007/978-1-4939-2383-0_11

Shah, M.H., Biradar, S. V, Paradkar, A.R., 2006. Spray dried glyceryl monooleate - magnesium trisilicate dry powder as cubic phase precursor. Int. J. Pharm. 323, 18-26. https://doi.org/10.1016/j.ijpharm.2006.05.040

Sou, T., Meeusen, E.N., de Veer, M., Morton, D.A. V, Kaminskas, L.M., McIntosh, M.P., 2011. New developments in dry powder pulmonary vaccine delivery. Trends Biotechnol. 29, 191-198. https://doi.org/10.1016/j.tibtech.2010.12.009

Speidel, K., Osen, W., Faath, S., Hilgert, I., Obst, R., Braspenning, J., Momburg, F., Hämmerling, G.J., Rammensee, H.-G., 1997. Priming of cytotoxic T lymphocytes by five heat-aggregated antigensin vivo: Conditions, efficiency, and relation to antibody responses. Eur. J. Immunol. 27, 2391-2399. https://doi.org/10.1002/eji.1830270938

Spicer, P., 2003. Cubosome Formation via Dilution: Kinetic Effects and Consumer Product Implications. ACS Symp. Ser. 861, 346-359.

Spicer, P.T., 2005. Progress in liquid crystalline dispersions: Cubosomes. Curr. Opin. Colloid Interface Sci. 10, 274-279. https://doi.org/10.1016/j.cocis.2005.09.004

Spicer, P.T., Hayden, K.L., Lynch, M.L., Ofori-Boateng, A., Burns, J.L., 2001. Novel process for producing cubic liquid crystalline nanoparticles (cubosomes). Langmuir 17, 5748-5756. https://doi.org/10.1021/la010161w

Spicer, P.T., Small, W.B., Lynch, M.L., Burns, J.L., 2002a. Dry powder precursors of cubic liquid crystalline nanoparticles (cubosomes). J. Nanoparticle Res. 4, 297-311. https://doi.org/10.1023/A:1021184216308

Spicer, P.T., Small, W.B., Lynch, M.L., Burns, J.L., 2002b. Dry powder precursors of cubic liquid crystalline nanoparticles (cubosomes). J. Nanoparticle Res. 4, 297-311. https://doi.org/10.1023/A:1021184216308

Storni, T., Kündig, T.M., Senti, G., Johansen, P., 2005. Immunity in response to particulate antigen-delivery systems. Adv. Drug Deliv. Rev. 57, 333-55. https://doi.org/10.1016/j.addr.2004.09.008

Uyama, M., Nakano, M., Yamashita, J., Hana, T., 2009. Useful modified cellulose polymers as new emulsifiers of cubosomes. Langmuir 25, 4336-4338. https://doi.org/10.1021/la900386q

White, K., Rades, T., Kearns, P., Toth, I., Hook, S., 2006. Immunogenicity of liposomes containing lipid core peptides and the adjuvant Quil A. Pharm. Res. 23, 1473-1481. https://doi.org/10.1007/s11095-0060272-z

Zhao, Z., Leong, K.W., 1996. Controlled delivery of antigens and adjuvants in vaccine development. J. Pharm. Sci. 85, 1261-70. https://doi.org/10.1021/js9602812 